\title{
A Comparison Of Functionality Between Mobile Apps And Browser-Based Applications
}

\author{
David Reavis, CCP, Ph.D., Texas A\&M University-Texarkana, USA
}

\begin{abstract}
The popularity of mobile apps is increasing at a rapid rate. The attraction for many users is the availability of software functionality on their mobile phone or tablet. One of the trade-offs with using a mobile app is the reduced functionality of the mobile app when compared with the functionality of a browser-based application. This study quantifies the level of functionality in both types of applications and compares five broad categories of mobile apps with their comparable browser-based applications to determine the amount of functionality that the user gives up when opting for the mobile app version of the software.
\end{abstract}

Keywords: Mobile App; Browser-Based Application; Functionality

\section{INTRODUCTION}

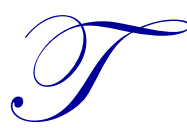

he literature regarding mobile apps contains numerous evaluations of the usability of mobile apps (Nayebi, Desharnais, \& Abran, 2012) (Kim, Oh, \& Moon, 2013). While considerable effort has been expended in measuring and evaluating usability, there has been far less investigation into the level of functionality available in mobile apps verses browser-based applications. The purpose of this study is to compare functionality in mobile apps with functionality in browser-based applications for the same use.

Intuitively, one would expect that the mobile version of a given software would do less than the browserbased version because of the smaller screen size, reliance on cell networks for much of the data transfer, and generally smaller available resources such as memory and processing power. But how much less functionality could the user expect? To quantify the actual functionality the researcher designed a study that compared mobile apps and the equivalent browser-based software. The comparison involved counting the number of function points for each application and grouping the results by categories to determine the level of functionality in each category as well as the aggregate comparison.

For a mobile user, there are multiple options available in accessing a given application. To illustrate, consider the popular social networking application Facebook. While a user is at their desktop computer, they could choose to access Facebook via their browser. While riding (not driving) in a car, the user might want to access Facebook via their cell phone app. Later that evening, the user might choose to use their iPad and access Facebook using either a browser or the mobile app. The organization delivering a software product must consider which of these delivery modes or paradigms best suit the product and which (one or all) should be pursued (Huy \& Thanh, 2012). In addition to the overall product delivery platform, an organization must also decide whether an app should be developed as a Native app and available on a specific platform such as Apple's App Store, or as a Mobile Web app which can be implemented in a mobile browser and do not require inclusion in any vendor's app delivery store (Seymour, Hussain, \& Reynolds, 2014). The Native app is developed for a specific target operating system and device and must be adapted to function on other mobile devices (Joorabchi, Mesbah, \& Kruchten, 2013). One of the complicating factors is the ability for some browser-based applications to dynamically format the presentation so that the elements on the page fit the display screen regardless of its size. This ability is called responsiveness. If a browser-based application is designed with responsive capabilities, it will adjust how information is displayed based 
on the size and resolution of the display screen on the device being used. As the researcher evaluated applications in the browser, the ability to adapt responsively was noted in the study and the impact of responsiveness will be discussed in the results below.

\section{RESEARCH DESIGN}

The categories for software in the study were Business, Entertainment, Food and Drink, Medical, and Social Networking. These categories are consistent with the categories on the App Store for Apple. The initial task for this study was to identify software that was available in both the mobile app format as well as the browser-based format for the given categories. Once these were selected the researcher accessed each application on an iPad for evaluation of the mobile app and on a chrome ${ }^{\circledR}$ browser for the browser-based software evaluation. The browser was set to a $100 \%$ zoom level on a monitor using landscape orientation at 1280 X 1024 resolution. In counting the function points for both apps and browser-based software, the researcher counted any link, list box, check box, radio button, input field, or other item that, when clicked or selected, would result in some action or response from the software. This included embedded features from advertisers, options to customize the display from the default, and a variety of unique selector controls found throughout the population of the study.

\section{LIMITATIONS OF THE STUDY}

There were some limitations for this study. One of the limiting factors involved the platform for evaluating the mobile apps. The researcher chose to use Apple's iPad for evaluating mobile apps and did not extend the study to other devices. While there are numerous considerations in selecting one mobile device over another (Rauch, 2011), the iPad is among the most popular and was deemed sufficient to represent the mobile app category. Another limitation was the availability of applications that offer both a browser-based application and a mobile app for the identical purpose. The researcher identified 45 such combinations and found that many of the apps currently available in Apple's App Store have companion Websites dedicated to the purpose of promoting the app and are not set up to duplicate the functions of the app. Another factor that was used to exclude several apps was a configuration that required a user log-in and significant user preference setup for the app and Website. For example, Pinterest requires that the user create a log-in and before proceeding to the home screen asks the user to identify several areas of interest. This profile has a direct impact on the number of function points displayed in the app and in the Browser interface and the variability introduced by the number of categories chosen would have caused excessive variation in the data. So sites that required this type of user customization were not included in the study. For certain applications, such as news sites or social media applications, the initial screen contained controls that would allow the user to scroll down and display hundreds or thousands of links. In these cases the researcher used the amount of data that was available in the initial page load and did not scroll down past the data loaded on the first page load. This technique had an impact on the results for the social networking category of results and is discussed below.

\section{RESULTS}

After evaluating the data for individual categories and for the aggregate of all categories, it is evident that for the majority of applications can be described such that on average the mobile app has about a quarter of the functionality of the companion Website. The exception to this is the social networking category, where the functionality averages just over 10 percent for the mobile apps. Table 1 lists the percent of functionality for each category of applications.

Table 1. Percent of Functionality of Mobile Apps Compared to Browser-based Application

\begin{tabular}{lcc}
\hline & Category & Percent of Functionality \\
\hline Business & $22.3 \%$ \\
Entertainment & $26.9 \%$ \\
Food and Drink & $28.2 \%$ \\
Medical & $21.6 \%$ \\
Social Networking & $10.8 \%$ \\
\hline
\end{tabular}




\section{Function Points}

For the population of the study, the average number of function points identified in the browser-based applications was 122 and the average number of function points in the mobile apps was 22 . This clearly shows that there is much more functionality available in the browser-based applications. The functionality of the browser-based applications depended on the design of the Website and was not easily grouped by category. Sites that tended to have larger numbers of links, such as news sites and social networking sites, were at the top of the functionality list because each link to a new story or post was counted as a function point.

\section{Social Networking}

Because of the design of the study and the near unlimited ability of social networking software to provide information on either platform, the usefulness of the data in determining functionality for this category may be less than the other categories. As an example, a Twitter user could conceivably scroll through thousands of tweets on either platform with each tweet providing options to reply, retweet, mark as a favorite, or other functions on every tweet. This could either be considered a duplication of functionality (each tweet has the same functionality) or added functionality for each tweet, therefore creating extremely high levels of functionality for the software. Dunn (2012) noted that for it is common for mobile apps to provide a relatively small number of functions which are used with great frequency. Social networking mobile apps are an example of this phenomenon because the majority of the data (in the example, tweets) occur with high frequency. The researcher chose to focus on the initial screen load for social media applications on each platform and this resulted in the $10.8 \%$ assessment of the mobile app over the browser-based application.

\section{Responsiveness}

Social Networking Websites were the category with the highest proportion of responsive behavior at $75 \%$ followed by Entertainment related Websites at $47 \%$. If the social networking applications are excluded from the dataset, the remaining categories exhibit a high level of correlation, .914 for the correlation coefficient between the percentage of sites that provide responsive behavior and the proportion of functionality from the browser-based application and the mobile app. From this correlation one could conclude that organizations that invest in responsive Website development are more likely to have increased functionality in a companion mobile app. Table 2 lists the percent of Websites in each category that exhibited responsive behavior.

Table 2. Percent of Functionality of Mobile Apps Compared to Browser-based Application

\begin{tabular}{lc}
\hline Category & Percent of Responsive Sites \\
\hline Business & $33.3 \%$ \\
Entertainment & $47.1 \%$ \\
Food and Drink & $42.9 \%$ \\
Medical & $33.3 \%$ \\
Social Networking & $75.0 \%$ \\
\hline
\end{tabular}

\section{IMPLICATIONS}

Users are willing to take advantage of mobile apps that provide far less functionality than the browserbased version of the same application. Considering the popularity explosion of mobile computing, the attraction and convenience of using certain software from a mobile device outweighs the increased functionality of a browserbased solution. The popularity of mobile apps is not a result of enhanced or even equivalent functionality, but because of key features that may be accessed from anywhere. Activities such as depositing a check into one's bank account, posting a picture of something to social media, and checking the weather in a user's current location or destination are all examples of some key features that draw users to mobile apps. In addition to the functionality for mobile apps, Nayebi (2012) identifies location awareness as a reason for mobile app adoption. The results of this study imply that developers should consider the most important features to the mobile user in developing mobile apps and that it is unnecessary to duplicate all of the functionality of a browser-based solution for the success of the mobile app. 
In the course of this study, the researcher noted some differences in the amount and content of selfpromotion and advertiser content between browser-based applications and mobile apps. A future study to quantify the differences in how related products and services are marketed in the mobile environment verses the browserbased environment may assist organizations in understanding marketing trends in the mobile environment.

\section{AUTHOR INFORMATION}

David Reavis teaches both Web-based and traditionally formatted classes in Management Information Systems and Management at Texas A\&M University-Texarkana. His work experience includes software development roles for manufacturing companies such as Cooper Tire and Rubber Co. and Alcoa. Reavis received his undergraduate BBA in computer information systems from Southern Arkansas University, his MBA from Texas A\&M UniversityTexarkana and his Ph.D. in information systems from Nova Southeastern University. Reavis research interests include mobile computing, electronic records management, and Web-based software development.

Email: David.reavis@tamut.edu

\section{REFERENCES}

Djamasbi, S., Kardzhaliyski, G. V., Liu, T., Gomez, W., \& Oglesby, F. E. (2013). App-Like Mobile Optimization and User Experience. Proceedings of the Twelfth Annual Workshop on HCI Research in MIS. Milan, Italy: Worcester Polytechnic Institute.

Dunn, B. K., Galletta, D. F., Hypolite, D., Puri, A., \& Raghuwanshi, S. (2012). Development of Smart Phone Usability Benchmarking Tasks. 46th Hawaii International Conference on System Sciences (HICSS) (pp. 1046-1052). Wailea, Maui, HI: IEEE.

Henze, N., \& Pielot, M. (2013, March/April). App Stores: External Validity for Mobile HCI. (R. a. Wakkary, Ed.) Interactions , 20 (2), pp. 33-38.

Huy, N. P., \& Thanh, D. V. (2012). Developing Apps for Mobile Phones. 7th Internatinal Conference on Computing and Convergence Technology (ICCCT) (pp. 907-912). Seoul: IEEE.

Huy, N. P., \& Thanh, D. V. (2012). Selecting the Right Mobile App Paradigms. (pp. 1-6). Taipei: IEEE Computer Society.

Joorabchi, M. E., Mesbah, A., \& Kruchten, P. (2013). Real Challenges in Mobile App Development. ACM / IEEE International Symposium on Emperical Software Engineering and Measurement (pp. 15-24). Vancouver, BC, Canada: University of British Columbia.

Kim, H. Y., Oh, J. S., \& Moon, H. N. (2013). Development of Smart Mobile App Assessment Model. 16th International Conference on Network-Based Information Systems (pp. 300-304). Gwangju: IEEE.

Nayebi, F., Desharnais, J., \& Abran, A. (2012). The State of the Art of Mobile Application Usability Evaluation. 25th IEEE Canadian Conference on Electrical and Computer Engineering (CCECE). Quebec, Canada: Universite du Quebec.

Rauch, M. (2011). Mobile Documentation: Usability Guidelines, and Considerations for Providing Documentation on Kindle, Tablets, and Smartphones. Professional Communication Conference (IPCC), 2011 IEEE International (pp. 1-13). Cincinnati, OH: IEEE.

Seymour, T., Hussain, J. Z., \& Reynolds, S. R. (2014). How to Create An App. International Journal of Management \& information Systems , 18 (2), 123-138. 